\title{
A MEAN ERGODIC THEOREM FOR FAMILIES OF CONTRACTIONS IN HILBERT SPACE
}

\author{
J. R. BLUM AND J. I. REICH
}

\begin{abstract}
Let $G$ be an LCA group and $h$ a Hilbert space, and let $T(g)$ be a function on $G$ into the contractions on $H$. Let $\left\{\sigma_{n}\right\}$ be a sequence of probability measures on $G$. Under suitable conditions on $T(g)$ and the sequence $\left\{\sigma_{n}\right\}$ we prove the strong convergence of the sequence $T_{n}$ $=\int T(g) \sigma_{n}(d g)$. In certain cases we identify the limiting operator.
\end{abstract}

Let $G$ be an LCA group and $\mathscr{B}(G)$ the Borel sets of $G$. $Q$ will denote the class of functions $l(\gamma)$ defined on $\hat{G}$ which are Fourier transforms of a probability measure defined on $\mathfrak{B}(G)$. If $\left\{\sigma_{n}\right\}_{n=1}^{\infty}$ is a sequence of such probability measures, with $\lim _{n} \sigma_{n}(\gamma)=l(\gamma)$ for $\forall \gamma \in \hat{G}$, we shall call the sequence $l(\gamma)$ amenable.

A set $S \in \mathscr{B}(G)$ is called amenable if $\exists$ a nonnegative Borel measure $\mu$ on $\mathscr{B}(G)$ and a sequence $\left\{S_{n}\right\}$ of Borel subsets of $S$ such that

(i) $\mu(B+g)=\mu(B)$ for every $B \in \mathscr{B}(G)$ and $g \in S$,

(ii) $0<\mu\left(S_{n}\right)<n=1,2, \ldots$,

(iii) $\lim _{n \rightarrow \infty} \mu\left[\left(S_{n}+g\right) \cap S_{n}\right] / \mu\left(S_{n}\right)=1$ for $\forall g \in S$.

If

$$
l_{S}(\gamma)=\left\{\begin{array}{ll}
1 \quad \text { if }\langle g, \gamma\rangle=1, \\
0 \text { otherwise, }
\end{array} \text { for } \forall g \in S,\right.
$$

then it is clear that $l_{S} \in \mathbb{Q}$ and, in fact, if $\sigma_{n}(\cdot)=\mu\left[S_{n} \cap(\cdot)\right] / \mu\left[S_{n}\right]$, then $\lim _{n} \hat{\sigma}_{n}(\gamma)=l_{S}(\gamma)$ for $\forall \gamma \in \hat{G}$.

Now let $T(g)$ be a function defined on $G$ whose values are contractions on a Hilbert space. We shall assume that $T(g)$ is positive definite. For a definition of a positive definite valued function, see Foias and Nagy [2, p. 25].

With these definitions we can state our main result.

TheOREM . Let $G$ be an LCA group and $H$ a Hilbert space. Let $T(g)$ be a positive definite function from $G$ to the bounded operators on $H$ such that $T(e)$ is the identity operator and $T(g)$ is weakly continuous. Then

(i) $T(g)$ is strongly continuous.

(ii) For a probability measure $\sigma$ on $G$ the integral $\int_{G} T(g) \sigma(d g)$ exists strongly and is a contraction operator on $H$.

Received by the editors March 12, 1975 and, in revised form, February 26, 1976.

AMS (MOS) subject classifications (1970). Primary 47A35, 47A20. 
(iii) With every function $l(\gamma) \in Q$ is associated a contraction operator $L^{l}$ on $H$ so that if $\left\{\sigma_{n}\right\}_{n=1}^{\infty}$ is an $l(\gamma)$-sequence of probability measures on $G$, then $L^{l}=$ strong- $\lim _{n \rightarrow \infty} \int_{G} T(g) \sigma_{n}(d g)$.

(iv) If $S$ is an amenable set and $T\left(g+g^{\prime}\right)=T(g) \cdot T\left(g^{\prime}\right)$ for $g, g^{\prime} \in S$, and if $\left\{\sigma_{n}\right\}_{n=1}^{\infty}$ is an $l_{S}(\gamma)$-sequence of probability measures on $G$, then strong- $\lim _{n \rightarrow \infty} \int_{G} T(g) \sigma_{n}(d g)=P_{\tilde{S}}$ where $P_{\tilde{S}}$ is the orthogonal projection onto the

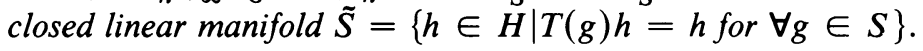

OUTLINE OF THE PROOF. The use of the dilation theorem in [2, p. 25] will be indicated by $(*)$.

(i) as defined in $(*)$ we have

$$
\left\|\left[T(g)-T\left(g^{\prime}\right)\right] h\right\|_{H}^{2}=\left(\left[U(g)-U\left(g^{\prime}\right)\right] h,\left[T(g)-T\left(g^{\prime}\right)\right] h\right)_{K} .
$$

Now use Schwartz's inequality and (*).

(ii) follows from (i).

(iii) Let $E(\cdot)$ be the resolution of the identity corresponding to $U(g)$ (as defined in (*)). Define for $l \in \mathbb{Q}: N^{l}=\int_{\hat{G}} l(\gamma) E(d \gamma)$; clearly $N^{l}$ is a contraction on $K$. Now let $L^{l}=P_{H} N^{l} ; P_{H}$ is the orthogonal projection onto $H$. If $\left\{\sigma_{n}\right\}_{n=1}^{\infty}$ is an $l(\gamma)$-sequence and $h \in H$, then

$$
\begin{aligned}
\left\|\int_{G} T(g) h \sigma_{n}(d g)-P_{H} N^{l} h\right\|^{2} & \\
= & \left(\int_{G} U(g) h \sigma_{n}(d g)-N^{l} h, \int_{G} T(g) h \sigma_{n}(d g)-P_{H} N^{l} h\right)_{K} \\
\leqslant & \leqslant \int_{G} U(g) h \sigma_{n}(d g)-N^{l} h\left\|_{K}^{2} \cdot 2 \cdot\right\| h \|_{H} .
\end{aligned}
$$

Now use the same technique as in the proof of $(*)$ to finish the proof.

(iv) We have to show that $L^{l_{S}}=P_{\tilde{S}}$ (using (iii)). Since $S$ is amenable the sequence of probability measures $\mu_{n}(\cdot)=\mu\left(S_{n} \cap(\cdot)\right) / \mu\left(S_{n}\right)$ is an $l_{S}$-sequence. Now let $h \in H$ and $f \in \tilde{S}$; then

$$
\begin{aligned}
\left(h-L^{l} h, f\right)_{H} & =\left(h-\lim _{n \rightarrow \infty} \int_{G} T(g) h \mu_{n}(d g), f\right)_{H} \\
& =(h, f)_{H}-\lim _{n \rightarrow \infty} \frac{1}{\mu\left(S_{n}\right)} \int_{S_{n}}\left(h, T^{*}(g) f\right)_{H} \mu(d g)=0 .
\end{aligned}
$$

Therefore $h-L^{l_{s}} h \in(\tilde{S})^{\perp}$. Now let $g^{\prime} \in S$; then

$$
\begin{aligned}
T\left(g^{\prime}\right) L^{l_{S}} h & =T\left(g^{\prime}\right) \underset{n \rightarrow \infty}{\text { strong- }} \lim \int_{G} T(g) h \mu_{n}(d g) \\
& =\lim _{n \rightarrow \infty} \frac{1}{\mu\left(S_{n}\right)} \int_{S_{n}} T\left(g^{\prime}\right) T(g) h \mu(d g) .
\end{aligned}
$$

Therefore, 


$$
\begin{aligned}
& L^{l_{s} h-T\left(g^{\prime}\right) L^{l_{s}} h} \\
& \quad=\lim _{n \rightarrow \infty}\left[\frac{1}{\mu\left(S_{n}\right)} \int_{S_{n}} T(g) h \mu(d g)-\frac{1}{\mu\left(S_{n}\right)} \int_{S_{n}} T\left(g^{\prime}+g\right) h \mu(d g)\right] \\
& =\lim _{n \rightarrow \infty} \frac{1}{\mu\left(S_{n}\right)} \int_{S_{n} \triangle\left(S_{n}+g^{\prime}\right)} T(g) h \mu(d g) .
\end{aligned}
$$

Therefore,

$$
\begin{aligned}
\left\|L^{l_{S}} h-T\left(g^{\prime}\right) L^{l_{s}} h\right\|_{H} & =\lim _{n \rightarrow \infty}\left\|\frac{1}{\mu\left(S_{n}\right)} \int_{S_{n} \triangle\left(S_{n}+g^{\prime}\right)} T(g) h \mu(d g)\right\|_{H} \\
& \leqslant \lim _{n \rightarrow \infty} \frac{\mu\left[S_{n} \triangle\left(S_{n}+g^{\prime}\right)\right]}{\mu\left(S_{n}\right)}\|h\|_{H}=0 .
\end{aligned}
$$

Therefore $L^{l_{s}} h \in \tilde{S}$ which together with $h-L^{l_{s}} h \in(\tilde{S})^{\perp}$ implies $L^{l_{s}}=P_{\tilde{S}}$. Q.E.D.

\section{REFERENCES}

1. J. R. Blum and B. Eisenberg, Generalized summing sequences and the mean ergodic theorem, Proc. Amer. Math. Soc. 42 (1974), 423-429. MR 48 \# 8749.

2. C. Foiaş and Béla Sz.-Nagy, Harmonic analysis of operators on Hilbert space, North-Holland, Amsterdam; American Elsevier, New York; Akadémiai Kiadó, Budapest, 1970. MR 43 \# 947.

Department of Mathematics, University of Wisconsin, Milwaukee, Wisconsin 53201 (Current address of J. I. Reich)

Current address (J. R. Blum): Mathematical Sciences Section, National Science Foundation, Washington, D.C. 20550 